\title{
ANEURYSM COMPLICATING TRAUMATIC SPONDYLOLISTHESIS
}

\author{
P. N. Robson and D. J. Tibbs, Newcastle upon Tyne, England \\ From the Royal Victoria Infirmary and University of Durham, Newcastle upon Tyne
}

A man aged twenty-six years was admitted to the Royal Victoria Infirmary two hours after a coal mining accident. He had been struck on the back and right side by a large mass of falling stonework. Immediately after the injury he had had pain in the back and in the left leg, but on admission the pain in the leg had been replaced by severe left-sided abdominal pain.

On examination the patient was pale and sweating; the blood pressure was $85 / 50$ millimetres $\mathrm{Hg}$. and the pulse rate 96 per minute. Multiple abrasions over the back indicated

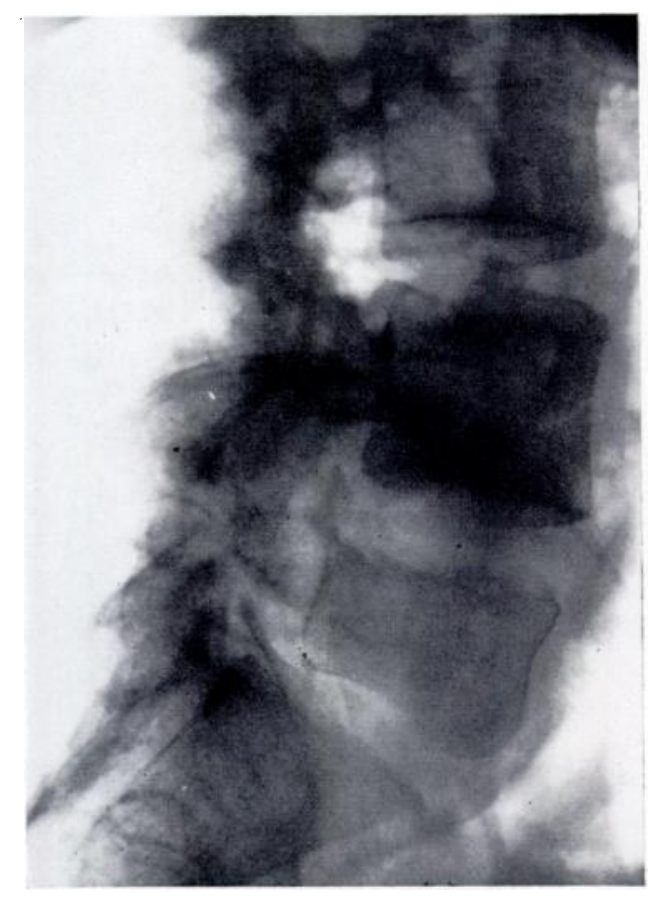

Fig. 1

Traumatic spondylolisthesis of the fifth lumbar vertebra on the first sacral.

where the stonework had struck him; the left loin was filled with a large fluctuant haematoma. No spinal movement was possible on account of severe pain, and there was tenderness over the lumbar spine with a distinct lateral shift of the fourth lumbar spinous process on the fifth. Neurological examination did not reveal any motor or sensory change in the limbs.

Radiographic examination-There were fractures of the transverse processes of all the lumbar vertebrae with extreme separation of the fragments on the left side. The body of the fifth lumbar vertebra was crushed, and there was slight lateral shift of the fourth lumbar vertebra on the fifth (Fig. 2). There were fractures through the pedicles of the fifth lumbar vertebra, with moderate spondylolisthesis (Fig. 1). No blood was detected in the urine.

Progress-The patient was given one pint of Dextran followed by four pints of blood. This brought his blood pressure up to $150 / 100$ millimetres $\mathrm{Hg}$. but the haemoglobin level two days later was only 60 per cent. 
During the next week the patient's condition gradually improved, and it was decided to immobilise the spine in a plaster bed for three months. At this time the swelling in the left loin had diminished and become soft, only a small bulge being visible.

In the second week the plaster bed was made but on his return to the ward after this the patient complained of severe pain in his back and down the medial aspect of the left thigh from the groin to the knee. On examination he was distressed and pale; the lumbar swelling was again tense and tender and had returned to its former dimensions. The blood pressure was 140/90 millimetres $\mathrm{Hg}$. and the pulse rate 100 per minute. Over the next three days the haematoma gradually became less tense and the pain subsided; at the end of this time the haemoglobin was 78 per cent.

In the third week the patient was placed into the plaster bed. At first he tolerated the immobilisation well, but after three days, while being turned to have the pressure areas treated, he complained of severe pain in the back. The following day he was taken out of the plaster bed as the tension of the haematoma made it impossible for him to remain in it with comfort.

By the sixth week the patient's condition was good, the haemoglobin being 92 per cent and the haematoma soft. It was decided to place him once more in the plaster bed, but the next day he had to be removed because he was complaining of pain in the back and of numbness and pain in the left thigh. This numbness persisted and evacuation of the haematoma was considered advisable.

First operation (seventh week)-A small oblique incision was made over the most prominent part of the haematoma in the loin. A large cavity was entered and from this one and a half pints of blood clot were removed. The wound was resutured without drainage and healed by first intention.

During the eighth week, while having physiotherapy to the legs, the patient complained of sudden severe pain in the back and weakness of the left leg. He also noticed that the haematoma had become larger and harder. On examination there were weakness of the quadriceps and sensory impairment in the distribution of the femoral nerve. Over the next two days the femoral nerve lesion became complete and the haematoma appeared even larger. The haemoglobin dropped to 76 per cent; platelet counts, clotting and bleeding times were within normal limits. Radiographs of the lumbar spine showed no increase in the vertebral displacement, but the fractured lumbar transverse processes on the left showed greatly increased separation (Fig. 2).

At this stage aortography was carried out because an aneurysm leaking intermittently was considered to be a real possibility. This failed to reveal any abnormality of the abdominal aorta or of the iliac arteries; the lumbar arteries however were not displayed. An excretion pyelograph performed at the same time is reproduced in Figure 2; it shows well the state of affairs, the whole loin being filled with haematoma grossly displacing the fractured transverse processes outwards and lifting the ureter forwards and inwards.

By now it was evident that the patient's life was being endangered by recurrent massive haemorrhage, for he looked pale and ill and was loosing weight steadily; the haemoglobin was 72 per cent and eight pints of blood had been given over the course of his illness. It was decided to explore the haematoma with full preparations to repair or remove an aneurysm of one of the main vessels.

Second operation (ninth week)-The haematoma was entered through a loin incision. A great mass of clot was disclosed and gently ladled out; in all five pints of soft clot were evacuated, and the walls of the cavity were composed of a further layer of organising thrombus over half an inch thick. During this process a firm, rounded swelling, adherent to its surroundings, was felt close to the lower lumbar spine; this was left undisturbed until the cavity had been completely cleared. Careful inspection showed that the swelling was about two inches across, and it could be seen and felt to pulsate vigorously. At this stage it was impossible to be certain from which vessel the aneurysm arose, and adequate exposure of the abdominal aorta and

vol. 39 B, NO. 3, AUGUST 1957 
left iliac vessels was clearly required before proceeding further; this was accomplished by extending the incision obliquely on to the lower abdomen and exposing the main vessels extraperitoneally. It was now seen that the anuerysm did not arise from these vessels, being separated from them by the psoas muscle. Compression of the lower abdominal aorta caused cessation of pulsation within the aneurysm, and it was concluded that it arose from a lumbar

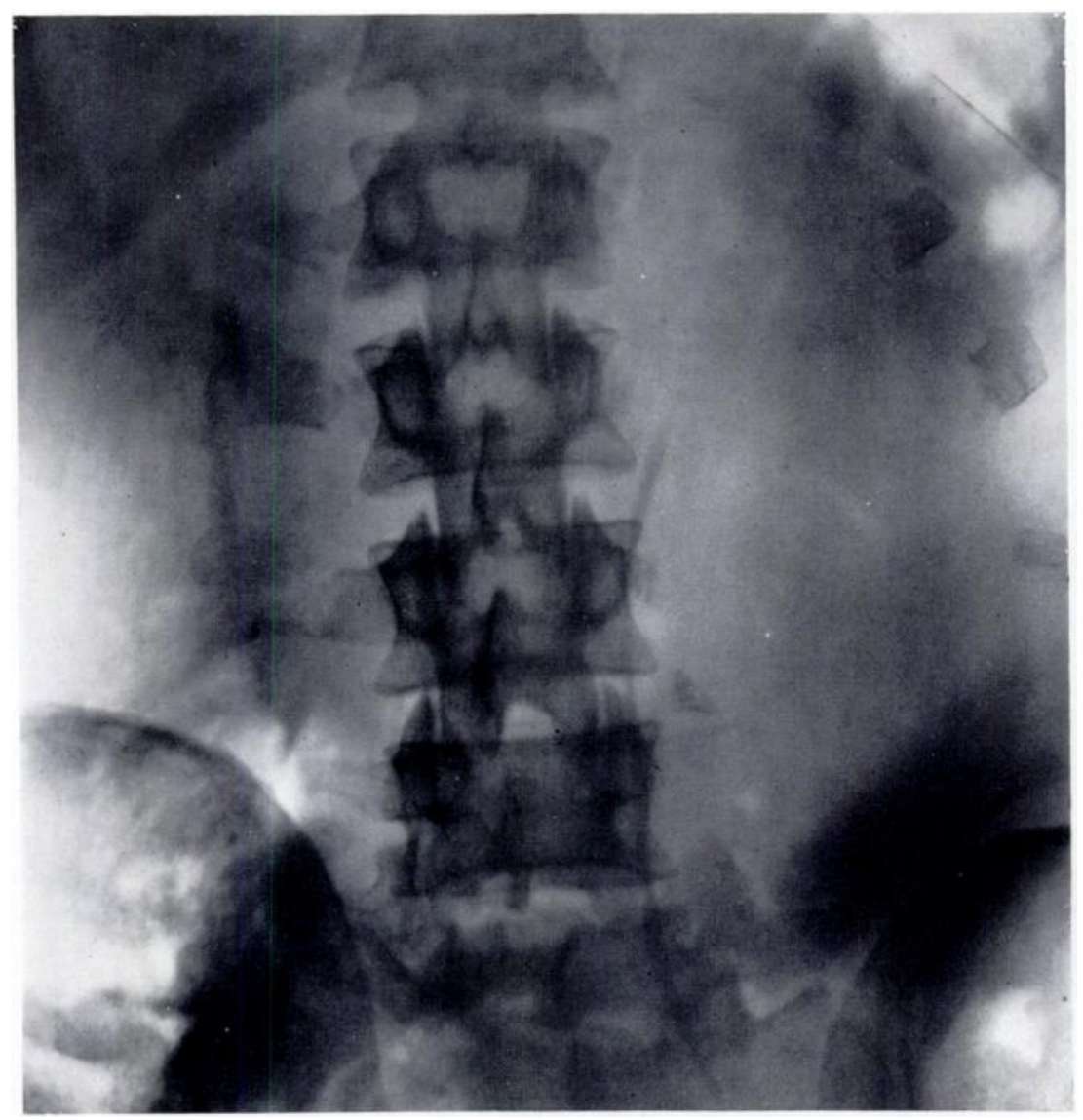

FIG. 2

Excretion pyelograph after aortography showing crushing of the fifth lumbar vertebra, extreme separation of the transverse processes, and displacement of the ureter.

artery. Cautious dissection of the aneurysm was begun, but almost at once heavy haemorrhage occurred, giving a clear demonstration of the mechanism of the recurrent haematoma formation that had characterised his illness. Pressure on the lower aorta enabled the dissection to be completed by peeling away the aneurysm until it was detached from its bed, leaving a posterior shell into which opened a moderate-sized artery that bled copiously when the aorta was released. The feeding artery was identified as the fourth lumbar and was readily controlled by two stitches encircling it. No distal outlet was observed and no further steps were taken to obliterate the aneurysm. A final inspection of the haematoma cavity was made: it was of considerable size, extending from the diaphragm above to the iliac fossa below, with the detached transverse processes projecting as sharp spurs from its lateral wall. A large drain was inserted into the cavity and the incision closed.

The aneurysm formed a rounded mass about two inches across and was composed of firm elot surrounding a comparatively small oval cavity half an inch in its longest diameter. Histological examination confirmed that the wall was thrombus only. 
The patient's condition improved dramatically after the operation. He regained good colour and felt much stronger in himself, gaining weight steadily. The wound healed well and recovery of the femoral nerve took place gradually over the next few weeks. A radiograph taken a month after the operation (Fig. 3) showed that the transverse processes had moved back closer to their origins presumably with contraction of the cavity.

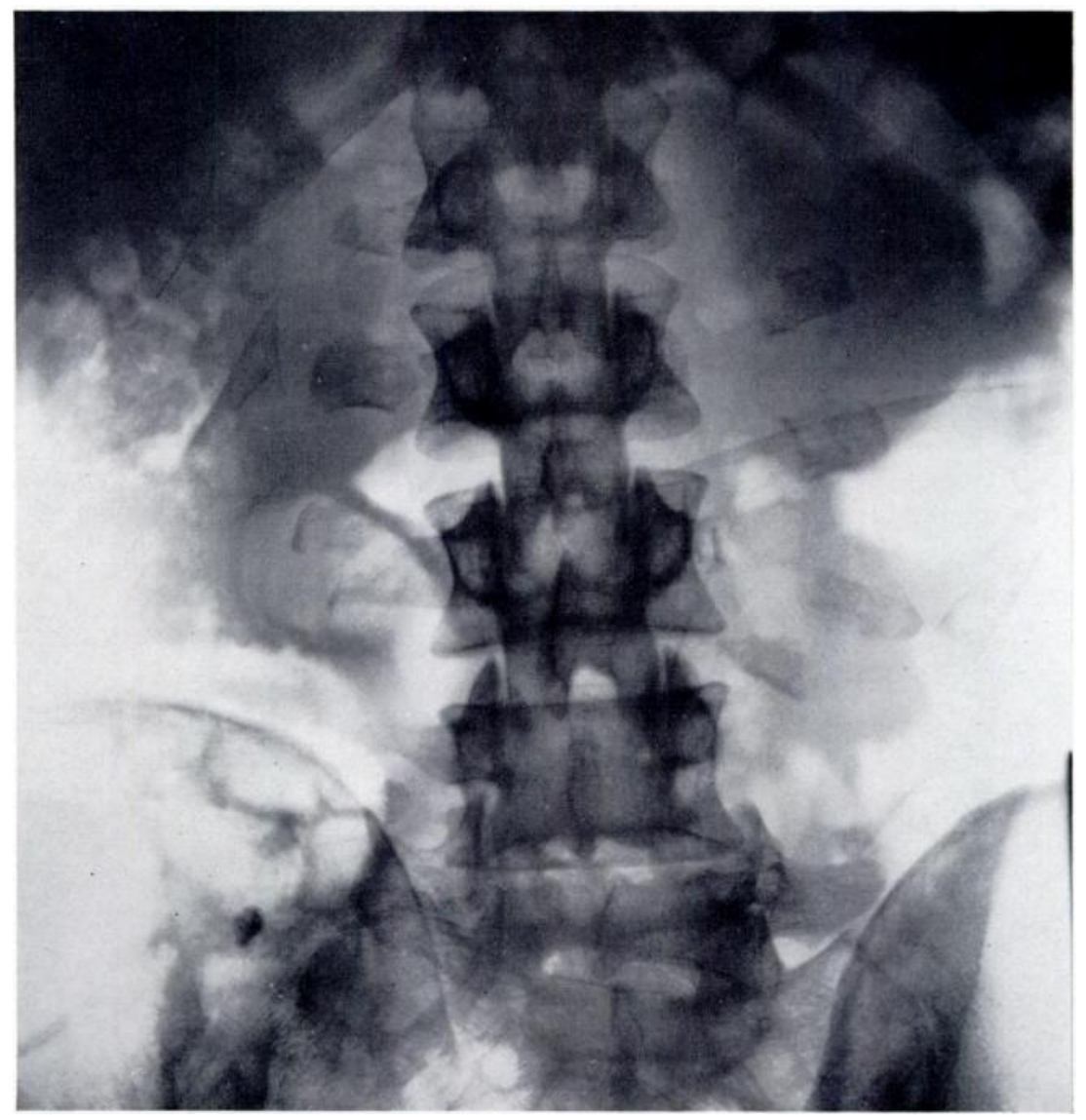

FIG. 3

One month after operation.

\section{COMMENT}

Traumatic spondylolisthesis is an uncommon injury, but it must be very rare for it to be associated with an aneurysm of a lumbar artery; our search of the literature failed to reveal a similar case. Presumably in our patient a severe closed injury caused laceration of a lumbar artery and this led to the formation of a false aneurysm which leaked intermittently when disturbed by any movement of neighbouring structures. Tears of this or similar arteries must be fairly common with closed injuries but in the great majority spontaneous sealing of the injured artery occurs so that there are no sequelae. Cases such as the one presented here are bound to arise occasionally and it is important that a recurrent massive haematoma should be regarded as a serious condition which may threaten the patient's life if the damaged vessel fails to close spontaneously. Exploration of the haematoma is a major undertaking, and preparations must be made for dealing with any possible arterial injury by resection and grafting should this prove necessary. 